\title{
Understanding the High Activity of Mildly Reduced Graphene Oxide Electrocatalysts in Oxygen Reduction to Hydrogen Peroxide
}

\author{
Xin Tan, ${ }^{\text {*a Hassan A. Tahini }}{ }^{\mathrm{a}}$ and Sean C. Smith ${ }^{* a}$ \\ a Integrated Materials Design Laboratory, Department of Applied Mathematics, Research School of Physics and Engineering, The \\ Australian National University, Canberra, ACT 2601, Australia. \\ *Email:Xin.Tan@anu.edu.au, Sean.Smith@anu.edu.au
}

The direct electrochemical synthesis of hydrogen peroxide $\left(\mathrm{H}_{2} \mathrm{O}_{2}\right)$ would provide an attractive alternative to the traditional anthraquinone oxidation process for continuous on-site applications. Its industrial viability depends greatly on developing cost-effective catalysts with high activity and selectivity. Recent experiments have demonstrated that mildly reduced graphene oxide (mrGO) electrocatalysts exhibit highly selective and stable $\mathrm{H}_{2} \mathrm{O}_{2}$ formation activity [e.g., H. W. Kim, M. B. Ross, N. Kornienko, L. Zhang, J. Guo, P. Yang and B. D. McCloskey, Nat. Catal., 2018, 1, 282-290]. However, the identification of active site structures for this catalytic process on mrGO is doubtful. Herein, by means of firstprinciples calculations, we examine the $\mathrm{H}_{2} \mathrm{O}_{2}$ formation activities of active site structures proposed in the experiments, and find that their activities are actually very low. Then, we systematically investigate the $\mathrm{H}_{2} \mathrm{O}_{2}$ formation activities of different oxygen functional group structures on mrGo based on experimental observations, and discover two types of oxygen functional group structures (2EP and 1ET+1EP) that have comparable or even lower overpotentials $\left(<0.10 \mathrm{~V}\right.$ ) for $\mathrm{H}_{2} \mathrm{O}_{2}$ formation compared with the state-of-the-art $\mathrm{PtHg}_{4}$ electrocatalyst. Our theoretical results reveal that the graphene edge and the synergetic effects between different oxygen functional groups are essential for the superior performance of mrGO for $\mathrm{H}_{2} \mathrm{O}_{2}$ production. This work not only provides a feasible explanation of the cause of high $\mathrm{H}_{2} \mathrm{O}_{2}$ formation activity of mrGO, but also offers a guide for the design, synthesis, and mechanistic investigation of advanced carbon-based electrocatalysts for effective $\mathrm{H}_{2} \mathrm{O}_{2}$ production.

\section{Introduction}

Hydrogen peroxide $\left(\mathrm{H}_{2} \mathrm{O}_{2}\right)$ is one of the 100 most important chemicals in the world with a wide range of applications, such as the paper and pulp, textile, and electronic industries, wastewater treatment, chemical oxidation (including the largescale production of propene oxide from propene oxidation) and others.[1-3] Its annual production exceeds 4 million tonnes and is expected to reach $\sim 6$ million tonnes in 2024, corresponding to US\$6.4 billion. [4] To date, the industrial synthesis of $\mathrm{H}_{2} \mathrm{O}_{2}$ still relies on the well established anthraquinone oxidation process, which is energy-intensive and is a multi-step method involving expensive palladium hydrogenation catalysts that generate substantial organic byproduct waste.[1] Furthermore, the transport, storage and handling of concentrated $\mathrm{H}_{2} \mathrm{O}_{2}$ are hazardous and expensive; therefore, on-site production of this chemical is highly desirable. [1] The direct synthesis of $\mathrm{H}_{2} \mathrm{O}_{2}$ from its constituent reagents $\left(\mathrm{H}_{2}\right.$ and $\left.\mathrm{O}_{2}\right)$ in the presence of $\mathrm{Pd}$-based catalysts provides a more effective and clean route.[5-7] However, the direct route needs to handle potentially explosive mixtures of $\mathrm{H}_{2}$ and $\mathrm{O}_{2}$, and satisfactory activity and desirable selectivity still remain formidable challenges.[5,8,9] Another attractive and alternative route for continuous on-site direct production of $\mathrm{H}_{2} \mathrm{O}_{2}$ is electrochemical route through the oxygen reduction reaction (ORR), using only $\mathrm{O}_{2}, \mathrm{H}_{2} \mathrm{O}$, and electricity as inputs with no waste released, which is safer, equally ecological and proceeds under mild conditions such as ambient temperature and pressure.[10-15] However, industrially viable electrochemical production of $\mathrm{H}_{2} \mathrm{O}_{2}$ requires the development of an electrocatalyst that is stable, cost-effective, active and

\section{Conceptual insights}

Carbon-based metal-free electrocatalysts have attracted intensive interest over the past several years as cost-effective alternatives to noble metals for the direct electrochemical synthesis of $\mathrm{H}_{2} \mathrm{O}_{2}$. Recent experimental investigations have shown that mildly reduced graphene oxide (mrGO) electrocatalysts is an efficient electrocatalyst for $\mathrm{H}_{2} \mathrm{O}_{2}$ production through two-electron oxygen reduction pathway, exceeding the performance of current state-of-the-art electrocatalysts. Nevertheless, the atomistic structures of active sites on mrGO, which are undoubtedly important for determining the reaction mechanism of the $\mathrm{H}_{2} \mathrm{O}_{2}$ production on $\mathrm{mrGO}$, and for the rational design of future catalysts, are not clear. To our best knowledge, the identification of active site structures for this catalytic process on mrGO, by means of firstprinciples calculations, has never been reported in the literature. Herein, for the first time, we systematically investigate the $\mathrm{H}_{2} \mathrm{O}_{2}$ formation activities of different oxygen functional group structures on $\mathrm{mrGO}$ based on experimental observations. Remarkably, two types of oxygen functional group structures (2EP and 1ET+1EP) are identified as the most active site structures with comparable or even lower overpotentials ( $<0.10 \mathrm{~V}$ ) for $\mathrm{H}_{2} \mathrm{O}_{2}$ formation compared with the state-of-the-art $\mathrm{PtHg}_{4}$ electrocatalyst. These theoretical results demonstrate that the graphene edge and the synergetic effects between different oxygen functional groups play a crucial role in the highly $\mathrm{H}_{2} \mathrm{O}_{2}$ formation activity of $\mathrm{mrGO}$, which provides a guide for the design, synthesis, and mechanistic investigation of advanced carbon-based electrocatalysts for effective $\mathrm{H}_{2} \mathrm{O}_{2}$ production.

selective for this reaction.

Numerous materials have been explored as potential electrocatalysts for the electrochemical production of $\mathrm{H}_{2} \mathrm{O}_{2}$, including noble metals and their alloys, such as $\mathrm{Pd}-\mathrm{Au},[16] \mathrm{Pt}-$ $\mathrm{Hg}[14]$ and $\mathrm{Pd}-\mathrm{Hg}$ [15], which require small overpotentials for $\mathrm{ORR}$ as well as high $\mathrm{H}_{2} \mathrm{O}_{2}$ selectivity (up to $~ 98 \%$ ). Of note, density functional theory (DFT) computations have been successfully applied for the rational design of the efficient 
electrocatalysts, and have found that $\mathrm{PtHg}_{4}$ alloys are the stateof-the-art electrocatalysts for the electrochemical production of $\mathrm{H}_{2} \mathrm{O}_{2}$ in acidic conditions. [14] However, the scarcity and, thus, high cost of noble metals limit their large-scale application. Carbon-based metal-free materials are promising as alternative catalysts for the electrochemical synthesis of $\mathrm{H}_{2} \mathrm{O}_{2}$ because of multiple advantages, including their high electronic conductivities, tunable molecular structures, earth-abundance, and electrochemically stable under reaction conditions. $[17,18]$ Very recently, the experimental results have shown that mildly reduced graphene oxide ( $\mathrm{mrGO}$ ) electrocatalysts exhibit highly selective and stable $\mathrm{H}_{2} \mathrm{O}_{2}$ formation activity at low overpotentials $(<0.10 \mathrm{~V})$, which exceed the performance of current state-of-the-art $\mathrm{PtHg}_{4}$ electrocatalysts.[19] By using spectroscopic structural characterization and in situ Raman spectroelectrochemistry, they have proposed that $s p^{2}$ hybridized carbon near epoxy (EP) group on unannealed mrGO basal plane and ring ether (ET) defects along annealed mrGO sheet edges are the most active sites for $\mathrm{H}_{2} \mathrm{O}_{2}$ production (see Fig. 3e in ref. [19]). However, the identification of the most active site structures for this catalytic process on $\mathrm{mrGO}$ is doubtful, which is undoubtedly important for determining the reaction mechanism of $\mathrm{H}_{2} \mathrm{O}_{2}$ production on $\mathrm{mrGO}$, and for the rational design of future catalysts. It is noteworthy that wellresolved DFT computations, especially when used in combination with experimental scattering and spectroscopic methods, have been proven as robust and powerful tools in exploring active site structures and reaction mechanisms in heterogeneous electrocatalysis. [20,21]

In this communication, we employ DFT computations to identify the active site structures for the electrochemical synthesis of $\mathrm{H}_{2} \mathrm{O}_{2}$ on mrGO. We examine the $\mathrm{H}_{2} \mathrm{O}_{2}$ formation activities of the active site structures proposed in the experiments, and find that their activities are actually very low. Remarkably, by calculating the $\mathrm{H}_{2} \mathrm{O}_{2}$ formation activities of different oxygen functional group structures on mrGO based on experimental observations, we discover two types of oxygen functional group structures (2EP and $1 E T+1 E P$ ) that have comparable or even lower overpotentials $(<0.10 \mathrm{~V})$ for $\mathrm{H}_{2} \mathrm{O}_{2}$ formation compared with the state-of-the-art $\mathrm{PtHg}_{4}$ electrocatalyst. These theoretical results demonstrate that the graphene edge and the synergetic effects between different oxygen functional groups play a crucial role in the highly $\mathrm{H}_{2} \mathrm{O}_{2}$ formation activity of $\mathrm{mrGO}$. Our findings not only provides a feasible explanation of the cause of high $\mathrm{H}_{2} \mathrm{O}_{2}$ formation activity of mrGO, but also offers a guide for the design, synthesis, and mechanistic investigation of advanced carbon-based electrocatalysts for effective $\mathrm{H}_{2} \mathrm{O}_{2}$ production.

\section{Results and discussion}

In electrochemical ORR, the $\mathrm{O}_{2}$ can be convert through one of two reactions: the 4-electron process to form $\mathrm{H}_{2} \mathrm{O}$ (equation (1)) or the 2-electron process to synthesize $\mathrm{H}_{2} \mathrm{O}_{2}$ (equation (2)). $[14,15]$

$$
\begin{array}{ll}
\mathrm{O}_{2}+4 \mathrm{H}^{+}+4 \mathrm{e}^{-} \rightarrow 2 \mathrm{H}_{2} \mathrm{O} & U^{0}=1.23 \mathrm{~V} \text { versus RHE } \\
\mathrm{O}_{2}+2 \mathrm{H}^{+}+2 \mathrm{e}^{-} \rightarrow \mathrm{H}_{2} \mathrm{O}_{2} & U^{0}=0.69 \text { V versus RHE }
\end{array}
$$

where $U^{0}$ is the equilibrium potential for each reaction, and RHE is the reversible hydrogen electrode. For an efficient catalyst for electroreduction of $\mathrm{O}_{2}$ to $\mathrm{H}_{2} \mathrm{O}_{2}$, the catalyst should provide high activity, by minimizing the overpotential for the 2-electron pathway (equation (1)), and high selectivity, by suppressing the 4-electron pathway (equation (2)).[14,15] In this work, we used the free energy of the sole adsorbed intermediate $\mathrm{HOO}^{*}$ $\left(\Delta G_{\mathrm{HOO}^{*}}\right)$, which is proven to be the key descriptor, to determine the catalytic activity and selectivity for electroreduction of $\mathrm{O}_{2}$ to $\mathrm{H}_{2} \mathrm{O}_{2}$.[14,15] This leads to a Sabatier volcano where the highest activity is achieved on the surface with a moderate interaction with $\mathrm{HOO}^{*}$. For the ideal catalyst, the $\Delta G_{\mathrm{HOO}^{*}}$ should be about $4.23 \mathrm{eV}$, so that the theoretical overpotential for $\mathrm{H}_{2} \mathrm{O}_{2}$ fomation $(\eta)$ should be zero. [14,15]

\section{The $\mathrm{H}_{2} \mathrm{O}_{2}$ formation activities of the active site structures on $\mathrm{mrGO}$ proposed in previous experiments}

In previous experimental works, EP group on unannealed $\mathrm{mrGO}$ basal plane and ring ET defects along annealed mrGO sheet edges have been proposed as highly active site structures towards $\mathrm{H}_{2} \mathrm{O}_{2}$ production, based only on spectroscopic structural characterization and in situ Raman spectroelectrochemistry.[19] To examine the $\mathrm{H}_{2} \mathrm{O}_{2}$ formation activities of these active site structures on $\mathrm{mrGO}$ by means of DFT computations, we calculated the $\Delta G_{\mathrm{HOO}^{*}}$ for these active site structures. Here, all $s p^{2}$-hybridized carbon near oxygen functional groups were considered as possible $\mathrm{HOO}^{*}$ adsorption sites, as shown in Fig. 1a and b. Our DFT results confirm that the carbon atoms in close proximity to oxygen functional groups are the most active adsorption sites for $\mathrm{HOO}^{*}$ (the optimized configurations are shown in Fig. $1 \mathrm{c}$ and d), where the overpotentials for $\mathrm{H}_{2} \mathrm{O}_{2}$ production are minimum. The computed values of $\Delta G_{\mathrm{HOO}^{*}}$ for these two structures are 5.15 and $5.18 \mathrm{eV}$, respectively, yielding high overpotentials of 0.92 and $0.95 \mathrm{~V}$, respectively, suggesting that these two active site structures on mrGO does not significantly contribute to the $\mathrm{H}_{2} \mathrm{O}_{2}$ production. Therefore, from a DFT computation point of view, neither EP group on mrGO basal plane nor ring ET defects along mrGO sheet edge is a highly active site structure towards $\mathrm{H}_{2} \mathrm{O}_{2}$ production, which is in contrast to previous experimental conclusion.[19] Two questions arise, how to explain the experimentally high activity of mrGO for electrochemical $\mathrm{H}_{2} \mathrm{O}_{2}$ production and what is the most active site structure for this catalytic process on mrGO? To address these questions, in the following, we systematically investigated the $\mathrm{H}_{2} \mathrm{O}_{2}$ formation activities of a wide range of oxygen functional group structures on mrGO based on experimental observations. 


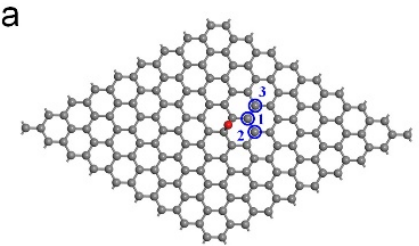

C

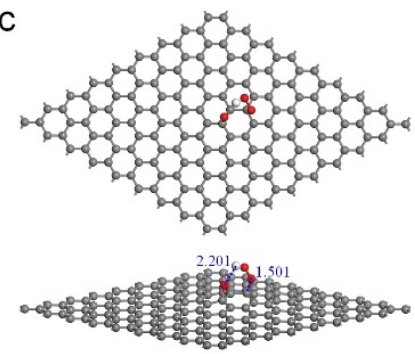

$\Delta G_{\mathrm{HOO}^{*}}=5.15 \mathrm{eV}$

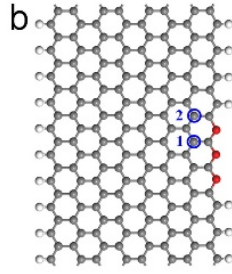

d

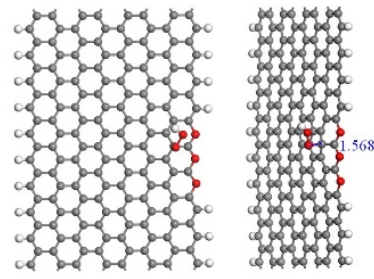

$\Delta G_{\mathrm{HOO}^{*}}=5.18 \mathrm{eV}$

Fig. 1 (a-b) Two oxygen functional group structures proposed in previous experiments as the highly active site structures towards $\mathrm{H}_{2} \mathrm{O}_{2}$ production (see Fig. 3e in ref. [19]). (a) The EP group on mrGO basal plane and (b) the ring ET defects along mrGO sheet edges. The $s p^{2}$-hybridized carbon near the oxygen functional groups denoted by blue circles are possible $\mathrm{HOO}^{*}$ adsorption sites considered in this study. Top and side view for the optimized configurations of $\mathrm{HOO}^{*}$ adsorbed on carbon in close proximity to (c) the EP group on mrGO basal plane and (d) the ring ET defects along mrGO sheet edges, with the minimum overpotential for $\mathrm{H}_{2} \mathrm{O}_{2}$ production. The grey, red and white balls represent $\mathrm{C}, \mathrm{O}$ and $\mathrm{H}$ atoms, respectively.

The $\mathrm{H}_{2} \mathrm{O}_{2}$ formation activities of highly active EP group structures on the unannealed mrGO

It has been demonstrated by previous experiments that EP group is the only oxygen functional group that contribute to the $\mathrm{H}_{2} \mathrm{O}_{2}$ production on the unannealed mrGO.[19] To identify the highly active site structures on the unannealed mrGO towards $\mathrm{H}_{2} \mathrm{O}_{2}$ production, we studied the $\mathrm{H}_{2} \mathrm{O}_{2}$ formation activities of different EP group structures.

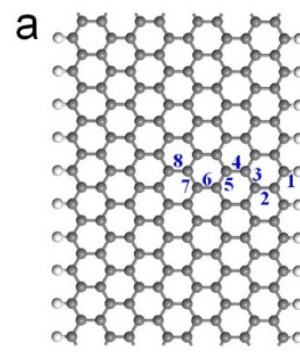

\begin{tabular}{|c|c|}
\hline Site & $E_{\mathbf{O}}^{F}(\mathbf{e V})$ \\
\hline 1 & 2.39 \\
\hline 2 & 3.69 \\
\hline 3 & 3.17 \\
\hline 4 & 3.56 \\
\hline 5 & 3.31 \\
\hline 6 & 3.51 \\
\hline 7 & 3.35 \\
\hline 8 & 3.50 \\
\hline
\end{tabular}
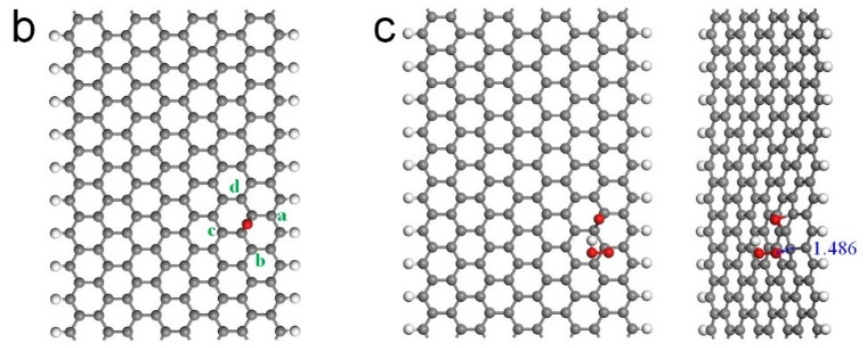

$\Delta G_{\mathrm{HOO}^{*}}=4.52 \mathrm{eV}$
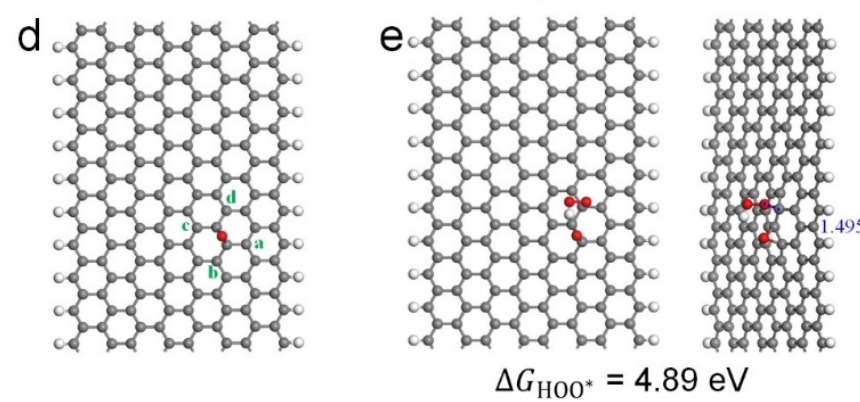

Fig. 2 (a) Structure of the 8-zGNRs with hydrogen-saturated edges used in our calculations. The blue numbers denote the possible adsorption sites for $\mathrm{O}$ adatom located close to $\mathrm{mrGO}$ sheet edge, and the corresponding $E_{\mathrm{O}}^{F}$ are listed in the table on the right. The optimized configurations of (b) the EP group located at sites 3 and (c) after the adsorption of HOO* $^{*}$ at the active site. The optimized configurations of (d) the EP group located at sites 5 and (e) after the adsorption of $\mathrm{HOO}^{*}$ at the active site. In (b) and (d), the green numbers denote possible adsorption sites for HOO*. The grey, red and white balls represent $\mathrm{C}, \mathrm{O}$ and $\mathrm{H}$ atoms, respectively.

Since the above DFT results proved that the EP group structure on mrGO basal plane has very poor activity for $\mathrm{H}_{2} \mathrm{O}_{2}$ production, we focused on EP group structures located close to $\mathrm{mrGO}$ sheet edge. To determine the energetically stability of EP group at different locations of the graphene, we calculated the formation energy of $O$ adatom, $E_{0}^{F}$, on graphene, which defined as $\quad E_{\mathrm{O}}^{F}=E($ graphene $+\mathrm{O})-E($ graphene $)-\left[E\left(\mathrm{H}_{2} \mathrm{O}\right)-\right.$ $\left.E\left(\mathrm{H}_{2}\right)\right]$, where $E$ (graphene $\left.+\mathrm{O}\right), E$ (graphene), $E\left(\mathrm{H}_{2} \mathrm{O}\right)$, and $E\left(\mathrm{H}_{2}\right)$ are the total energy of graphene with $\mathrm{O}$ adatom, bare graphene, $\mathrm{H}_{2} \mathrm{O}$ gas, and $\mathrm{H}_{2}$ gas, respectively. According to this definition, a less positive formation energy indicates a more energetically stable structure. Fig. 2a shows possible adsorption sites for $\mathrm{O}$ adatom located close to $\mathrm{mrGO}$ sheet edges, and the corresponding $E_{\mathrm{O}}^{F}$ are listed in the table on the right. Except for site 1 , where the $O$ adatom site on top of carbon atom, a surface EP group consists of $\mathrm{O}$ adatom above a bright $\mathrm{C}-\mathrm{C}$ site is the 
most stable configuration of $\mathrm{O}$ adatom located close to $\mathrm{mrGO}$ sheet edge. Since we focused on the EP group structures on graphene, $\mathrm{O}$ adsorbed on site 1 was not considered here. Interestingly, the $E_{\mathrm{O}}^{F}$ of EP group located at site 3 (Fig. 2b) and site 5 (Fig. 2d) are 3.17 and $3.31 \mathrm{eV}$, respectively, which are more energetically stable (less positive) than that for the EP group on mrGO basal plane $\left(E_{\mathrm{O}}^{F}=3.40 \mathrm{eV}\right)$, indicating that the EP group prefer to locate close to $\mathrm{mrGO}$ sheet edges.

In order to calculate the $\mathrm{H}_{2} \mathrm{O}_{2}$ formation activities of the EP group structures located at the stable sites close to $\mathrm{mrGO}$ sheet edges, i.e. at site 3 and site 5 , all carbon atoms in close proximity to the EP group were considered as possible $\mathrm{HOO}^{*}$ adsorption sites, as shown in Fig. $2 \mathrm{~b}$ and $\mathrm{d}$. The computed values of $\Delta G_{\mathrm{HOO}^{*}}$ for the EP group at site 3 and site 5 with HOO* adsorbed at the active site (the optimized configurations are shown in Fig. 2c and e) are 4.52 and $4.89 \mathrm{eV}$, respectively, corresponding to the overpotentials of 0.29 and $0.66 \mathrm{~V}$, respectively. These results indicate that the EP group structures located close to mrGO sheet edges are more active towards $\mathrm{H}_{2} \mathrm{O}_{2}$ production in comparison with the EP group structure on $\mathrm{mrGO}$ basal plane $(\eta=0.92 \mathrm{~V})$. Of note, the overpotentials of these structures are still quite high compared to that of the state-of-the-art $\mathrm{PtHg}_{4}$ electrocatalyst $(\eta \approx 0.10 \mathrm{~V})$, suggesting these structures are also not highly active structures for $\mathrm{H}_{2} \mathrm{O}_{2}$ production.

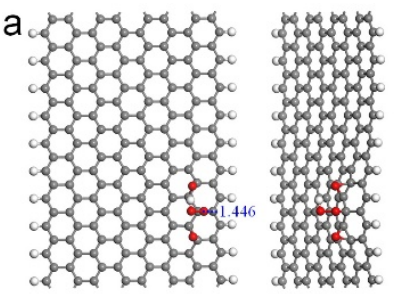

$\Delta G_{\mathrm{HOO}^{*}}=4.09 \mathrm{eV}$

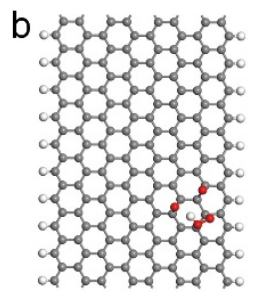

$$
\Delta G_{\mathrm{HOO}^{*}}=4.30 \mathrm{eV}
$$

Fig. 3 Top and side view for the optimized configurations of (a) two neighbouring EP groups at site 3 , and (b) one EP group at site 3 and neighbouring EP group at site 5, after the adsorption of $\mathrm{HOO}^{*}$ at the active site. The grey, red and white balls represent $\mathrm{C}, \mathrm{O}$ and $\mathrm{H}$ atoms, respectively.

Previous studies have established that the synergetic effects between functional groups may significantly affect the catalytic activity of graphene-based electrocatalyst.[22,23] Encouraged by the above graphene edge effects on the enhancement of $\mathrm{H}_{2} \mathrm{O}_{2}$ formation activities of the EP group structures, we investigated the $\mathrm{H}_{2} \mathrm{O}_{2}$ formation activity of the structures consist of two neighbouring EP groups (2EP) located close to mrGO sheet edges. Here, we considered two structures, including two neighbouring EP groups at site 3, and one EP group at site 3 and neighbouring EP group at site 5, and the optimized configurations of these two structures after the adsorption of $\mathrm{HOO}^{*}$ at the active site are shown in Fig. 3a and b. The computed values of $\Delta G_{\mathrm{HOO}^{*}}$ for these two $2 \mathrm{EP}$ structures are 4.09 and $4.30 \mathrm{eV}$, respectively, yielding small overpotentials of 0.14 and $0.07 \mathrm{~V}$, respectively. These values are comparable with the current state-of-the-art $\mathrm{PtHg}_{4}$ electrocatalyst $(\eta \approx$ $0.10 \mathrm{~V}$ ), suggesting these 2EP structures are highly active for $\mathrm{H}_{2} \mathrm{O}_{2}$ production, which are in good agreement with the experimental observations that the unannealed mrGO with EP groups exhibit highly $\mathrm{H}_{2} \mathrm{O}_{2}$ formation activity at low overpotentials (<0.10 V).[19]

\section{The $\mathrm{H}_{2} \mathrm{O}_{2}$ formation activities of highly active structures consist of} ring ET and EP groups on the annealed mrGO

Now, we shifted our attention to active site structures on the annealed mrGO. Previous experiments have provided strong evidence that the $\mathrm{mrGO}$ thermal treatment at $600{ }^{\circ} \mathrm{C}$ produces ring ET groups at mrGO sheet edges,[19,24,25] resulting in higher $\mathrm{H}_{2} \mathrm{O}_{2}$ formation activity compared with unannealed mrGO.[19] In order to explain the experimental observations and identify highly active structures on the annealed $\mathrm{mrGO}$ towards $\mathrm{H}_{2} \mathrm{O}_{2}$ production, we studied the $\mathrm{H}_{2} \mathrm{O}_{2}$ formation activity of different oxygen functional group structures containing ring ET group along mrGO sheet edge. First, we considered a single ring ET group located along $\mathrm{mrGO}$ sheet edge (Fig. 4a). The computed value of $\Delta G_{\mathrm{HOO}^{*}}$ for this structure after the adsorption of $\mathrm{HOO}^{*}$ at the active site (the optimized configuration is shown in Fig. $4 \mathrm{~b})$ is $4.63 \mathrm{eV}(\eta=0.40 \mathrm{~V})$, which is relatively high compared with the state-of-the-art $\mathrm{PtHg}_{4}$ electrocatalyst $(\eta \approx 0.10 \mathrm{~V})$. This implies that the single ring ET group located along mrGO sheet edge is not highly active structure for $\mathrm{H}_{2} \mathrm{O}_{2}$ production.

Synergetic effects between different functional groups have been shown to play an important role in adjusting the catalytic activity of graphene-based electrocatalyst.[22,23] EP group is the most likely oxygen functional group that might interact with the ring ET group along mrGO sheet edge because of the high stability of the EP group located close mrGO sheet edge, which has been confirmed by our DFT calculations. For this purpose, we studied the $\mathrm{H}_{2} \mathrm{O}_{2}$ formation activities of different oxygen functional group structures consist of a ring ET group and an EP group along $\mathrm{mrGO}$ sheet edge (1ET+1EP). Here, we considered all energetically stable sites for EP group close to $\mathrm{mrGO}$ sheet edge (in Fig. 2a), and constructed the structures by adding an EP group at one of the energetically stable sites near to the ring ET group along $\mathrm{mrGO}$ sheet edge. Based on our DFT calculations of the $\Delta G_{\mathrm{HOO}^{*}}$ for different 1ET+1EP structures, we identified two highly active $1 \mathrm{ET}+1 \mathrm{EP}$ structures that exhibit superior $\mathrm{H}_{2} \mathrm{O}_{2}$ formation activity, as shown in Fig $4 \mathrm{c}$ and e. The computed values of $\Delta G_{\mathrm{HOO}^{*}}$ for these two structures after the adsorption of $\mathrm{HOO}^{*}$ at the active site (the optimized configurations are shown in Fig. 4d and e) are 4.23 and $4.20 \mathrm{eV}$, respectively, corresponding negligible overpotentials of less than $0.03 \mathrm{~V}$, which are even lower than that for the state-of-the-art $\mathrm{PtHg}_{4}$ electrocatalyst $(\eta \approx 0.10 \mathrm{~V}$ ). Our DFT calculations predict that these $1 \mathrm{ET}+1 \mathrm{EP}$ structures are the most active site structures on the annealed mrGO, and their $\mathrm{H}_{2} \mathrm{O}_{2}$ formation activities are even higher than that of the state-of-the-art $\mathrm{PtHg}_{4}$ electrocatalyst. These results are in good agreement with the experimental observations that the $\mathrm{H}_{2} \mathrm{O}_{2}$ formation activities of the annealed mrGO with ET and EP groups are higher than that of the unannealed $\mathrm{mrGO}$, which exceed the performance of the state-of-the-art $\mathrm{PtHg}_{4}$ electrocatalyst.[19] 


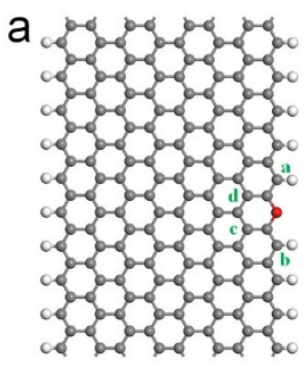

b
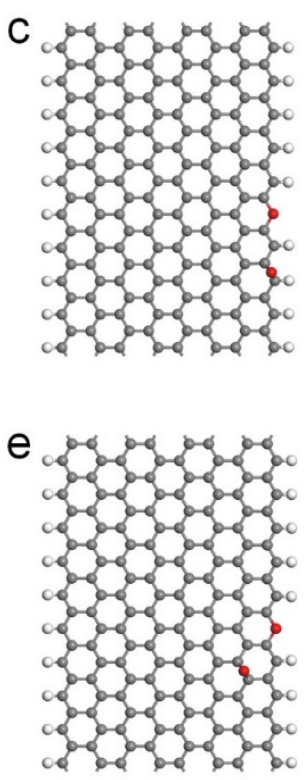

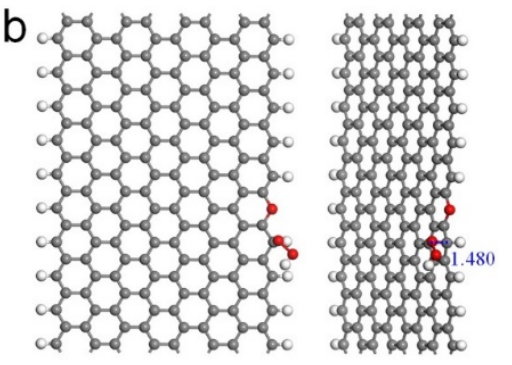

$\Delta G_{\mathrm{HOO}^{*}}=4.63 \mathrm{eV}$

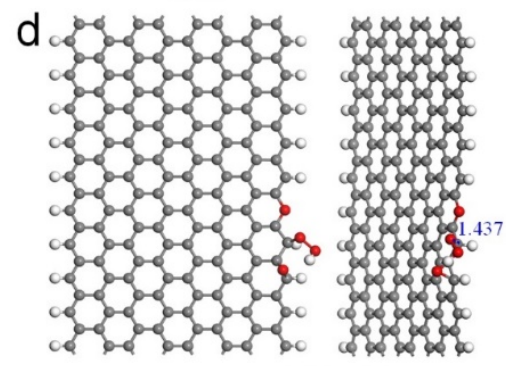

$\Delta G_{\mathrm{HOO}^{*}}=4.23 \mathrm{eV}$

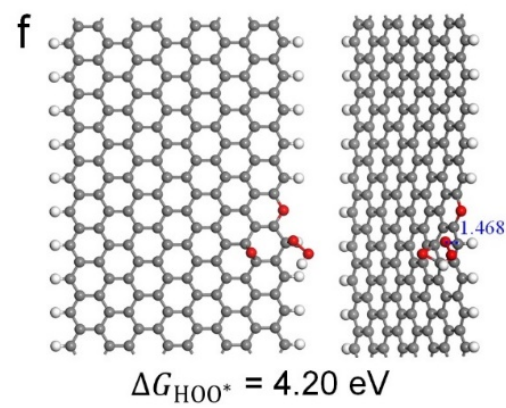

Fig. 4 The optimized configurations of (a) a single ring ET group located along $\mathrm{mrGO}$ sheet edge, and (b) after the adsorption of $\mathrm{HOO}^{*}$ at the active site. The green numbers denote the possible adsorption sites for $\mathrm{HOO}^{*}$ adsorption. The optimized configurations of (c, e) two most active 1ET+1EP structures, and (d, f) after the adsorption of $\mathrm{HOO}^{*}$ at the active site. The grey, red and white balls represent $\mathrm{C}, \mathrm{O}$ and $\mathrm{H}$ atoms, respectively.

\section{Theoretically calculated $\mathrm{H}_{2} \mathrm{O}_{2}$ formation activities of different oxygen functional group structures on $\mathrm{mrGO}$}

In order to clearly compare and further understand the $\mathrm{H}_{2} \mathrm{O}_{2}$ formation activities of different oxygen functional group structures on $\mathrm{mrGO}$, we summarized our DFT calculation results in a Sabatier volcano plot of limiting potential $\left(U_{\mathrm{L}}\right)$ versus $\Delta G_{\mathrm{HOO}^{*}}$, as shown in Fig. 5 . From this figure, we can draw two conclusions: 1. The oxygen functional groups (EP or ET groups) located close to $\mathrm{mrGO}$ sheet edges can significantly enhance the $\mathrm{H}_{2} \mathrm{O}_{2}$ formation activity ( $\eta$ for 1EP and 1ET are 0.29 and $0.40 \mathrm{~V}$, respectively) in comparison with oxygen functional group on mrGO basal plane $(\eta=0.92 \mathrm{~V})$, indicating the graphene edge effect is an important factor for the $\mathrm{H}_{2} \mathrm{O}_{2}$ formation activity of mrGO. 2. The synergetic effects between different oxygen functional groups can further increase the $\mathrm{H}_{2} \mathrm{O}_{2}$ formation activity of mrGO. For example, $\eta$ for 2 EP structures $(<0.10 \mathrm{~V})$ are comparable with the state-of-the-art $\mathrm{PtHg}_{4}$ electrocatalyst, and $\eta$ for $1 \mathrm{ET}+1 \mathrm{EP}$ structures are less than $0.03 \mathrm{~V}$, which reach the top of volcano plot and exceed the state-of-the-art $\mathrm{PtHg}_{4}$ electrocatalyst. To further understand the $\mathrm{H}_{2} \mathrm{O}_{2}$ formation activities of different oxygen functional group structures on $\mathrm{mrGO}$, we calculated charge densities of the active sites, i. e. the carbon atoms where HOO* stick to, for different oxygen functional group structures on $\mathrm{mrGO}$, and compared with 8zGNRs with no oxygen functional group (bare-G), as shown in Fig. S1 in ESI. The calculation results clearly show that the electrons on active sites follow the order of $2 E P>1 E P>$ bare- $G$ and $1 E T+1 E P>1 E T>$ bare- $G$, which indicate that more electrons in $2 \mathrm{EP}$ and $1 \mathrm{ET}+1 \mathrm{EP}$ structures can be donated to adsorbed $\mathrm{HOO}^{*}$, and lead to the stronger $\mathrm{HOO}^{*}$ adsorption, i.e. the enhanced $\mathrm{H}_{2} \mathrm{O}_{2}$ formation activities, compared to that of bareG. Overall, our theoretical results reveal that the graphene edge and the synergetic effects between different oxygen functional groups are essential for the superior performance of mrGO for $\mathrm{H}_{2} \mathrm{O}_{2}$ production.

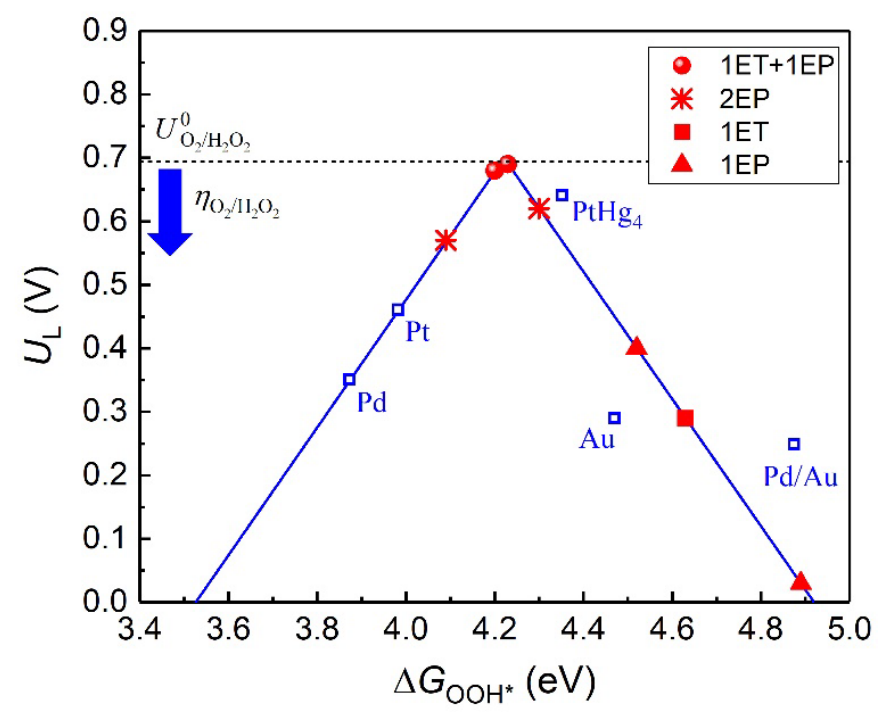

Fig. 5 The calculated $U_{\mathrm{L}}$ for $\mathrm{H}_{2} \mathrm{O}_{2}$ production of various oxygen functional group structures as a function of $\Delta G_{\mathrm{HOO}^{*}}$. The blue solid lines represent the theoretical Sabatier volcano. $[14,15,26]$ The empty squares are the data adapted from ref. [14], and $\mathrm{PtHg}_{4}$ is the reported state-of-the-art electrocatalyst with $\eta \approx$ $0.10 \mathrm{~V}$.[14] The equilibrium potential for $\mathrm{H}_{2} \mathrm{O}_{2}$ production, $U_{\mathrm{O}_{2} / \mathrm{H}_{2} \mathrm{O}_{2}}^{0}=0.69 \mathrm{~V}$, is shown as the dotted line. 1EP and $1 \mathrm{ET}$ denote a single EP group and a single ring ET group located close to $\mathrm{mrGO}$ sheet edge, respectively. 2EP denote the structures with two EP groups located close to mrGO sheet edge. 1ET+1EP denotes the structures consist of an EP group and an ET group located close to mrGO sheet edge. Please note that the calculation results for the active site structures proposed in previous experiments[19] are out of the range.

\section{Conclusions}

In conclusion, based on first-principles calculations, we have examined the $\mathrm{H}_{2} \mathrm{O}_{2}$ formation activities of the active site structures proposed in the experiments, and found that their 
activities are actually very low. To explain the experimentally high activity of mrGO for electrochemical $\mathrm{H}_{2} \mathrm{O}_{2}$ production and identify the most active site structures for this catalytic process on mrGO, we have systematically investigated the $\mathrm{H}_{2} \mathrm{O}_{2}$ formation activities of different oxygen functional group structures on mrGO based on experimental observations, and discovered two types of oxygen functional group structures (2EP and 1ET+1EP) that have high $\mathrm{H}_{2} \mathrm{O}_{2}$ formation activities. 2EP structures have comparable $\eta(<0.10 \mathrm{~V})$ with the state-of-theart $\mathrm{PtHg}_{4}$ electrocatalyst, and 1ET+1EP structures have even lower $\eta(<0.03 \mathrm{~V})$ than that for the state-of-the-art $\mathrm{PtHg}_{4}$ electrocatalyst, which are in good agreement with the experimental observations that the $\mathrm{H}_{2} \mathrm{O}_{2}$ formation activities of the annealed $\mathrm{mrGO}$ with ET and EP groups are higher than that of the unannealed mrGO with EP groups. Our theoretical results reveal that the graphene edge and the synergetic effects between different oxygen functional groups are essential for the superior performance of $\mathrm{mrGO}$ for $\mathrm{H}_{2} \mathrm{O}_{2}$ production. This work not only provides a feasible explanation of the cause of high $\mathrm{H}_{2} \mathrm{O}_{2}$ formation activity of $\mathrm{mrGO}$, but also offers a guide for the design, synthesis, and mechanistic investigation of advanced carbon-based electrocatalysts for effective $\mathrm{H}_{2} \mathrm{O}_{2}$ production.

\section{Computational methods}

Our DFT calculations were carried out using the linear combination of atomic orbital and spin-unrestricted method implemented in $\mathrm{Dmol}^{3}$ package.[27] The generalized gradient approximation (GGA) in Perdew-Burke-Ernzerhof (PBE) functional form[28] together with an all-electron double numerical basis set with polarization function (DNP) were adopted. Considering the standard PBE functional is incapable of giving an accurate description of weak interactions, we adopted a DFT+D (D stands for dispersion) approach with the Grimme vdW correction in our computations.[29] The realspace global cutoff radius was set to be $5.5 \AA$.

In order to simulate graphene basal plane and graphene sheet edge, we considered graphene monolayer and zigzag graphene nanoribbons (zGNRs), respectively, as shown in Fig. 1a and $b$. For graphene monolayer, a $9 \times 9$ supercell was used, and the vacuum space was set as $15 \AA$ in the $z$ direction to avoid interactions between periodic images. For zGNRs, we used 8zGNRs with hydrogen-saturated edges.[30] The supercell size was $30.7 \times 24.6 \times 15.0 \AA$, and the periodic boundary conditions were applied with the vacuum separation distance set to 13 and $15 \AA$ along the $x$ and $z$ directions, respectively, to avoid interaction between two ribbons. The Brillouin zone was sampled with a $3 \times 3 \times 1$ and $1 \times 2 \times 1 \Gamma$ centered $k$ points grid[31] for graphene monolayer and zGNRs, respectively. In geometry optimizations, all the atomic coordinates were fully relaxed up to the residual atomic forces smaller than $0.002 \mathrm{Ha} / \AA$, and the total energy was converged to $10^{-5} \mathrm{Ha}$. On the basis of previous experimental observations, two types of oxygen functional groups, EP group on the basal plane and ring ET group along the sheet edges, are closely related to the catalytic process on the mrGO.[19] Here we added those oxygen functional groups in our simulation models to model the mrGO.
It is generally accepted that the electroreduction of $\mathrm{O}_{2}$ to $\mathrm{H}_{2} \mathrm{O}_{2}$ involves two coupled electron and proton transfers:[14,15]

$$
\begin{aligned}
& \mathrm{O}_{2}+^{*}+\left(\mathrm{H}^{+}+\mathrm{e}^{-}\right) \rightarrow \mathrm{HOO}^{*} \\
& \text { HOO }^{*}+\left(\mathrm{H}^{+}+\mathrm{e}^{-}\right) \rightarrow \mathrm{H}_{2} \mathrm{O}_{2}+^{*}
\end{aligned}
$$

where ${ }^{*}$ represents an unoccupied active site on electrocatalyst surface, and $\mathrm{HOO}^{*}$ represents the sole adsorbed intermediate for the reaction. The $\Delta G_{\mathrm{HOO}^{*}}$ is proven to be the key parameter, or descriptor, to characterize the activity for electroreduction of $\mathrm{O}_{2}$ to $\mathrm{H}_{2} \mathrm{O}_{2}$, which lead to a Sabatier volcano where the highest activity is achieved on the surface with a moderate interaction with $\mathrm{HOO}^{*}$. $[14,15]$ The $U_{\mathrm{L}}$ is defined as the lowest potential at which the two reaction steps (equation (3) and equation (4)) are downhill in free energy, and $\eta$ is defined as the maximum difference between the $U_{\mathrm{L}}$ and $U^{0}$. For the ideal catalyst, the $\Delta G_{\mathrm{HOO}^{*}}$ should be about $4.23 \mathrm{eV}$, so that $\eta=0 \mathrm{~V} .[14,15]$ Weaker binding to $\mathrm{HOO}^{*}$ (right-hand side of volcano) are limited by hydrogenation of $\mathrm{O}_{2}$ (equation (3)), while stronger binding to $\mathrm{HOO}^{*}$ (left-hand side of volcano) will lead to the overpotential due to the reduction of $\mathrm{HOO}^{*}$ to $\mathrm{H}_{2} \mathrm{O}_{2}$ (equation (4)). [14,15]

The $\Delta G_{\mathrm{HOO}^{*}}$ was calculated based on a computational hydrogen electrode (CHE) model proposed by Nørskov et al..[32,33] In this model, the free energy changes at each electrochemical step involving an proton-electron transfer are calculated using the definition that the free energy of $\left(\mathrm{H}^{+}+\mathrm{e}^{-}\right)$equals to $\frac{1}{2} \mathrm{H}_{2}(g)$ for standard hydrogen electrode (SHE). According to this method, the $\Delta G_{\mathrm{HOO}^{*}}$ was calculated as:[14,33]

$$
\Delta G_{\mathrm{HOO}^{*}}=\Delta E_{\mathrm{HOO}^{*}}+\Delta \mathrm{ZPE}-T \Delta S
$$

where $\Delta E_{\mathrm{HOO}^{*}}$ is the binding energies of $\mathrm{HOO}^{*}$ directly obtained from DFT calculations, ZPE is the zero-point energy, $T$ is temperature, and $S$ is the entropy. Here, zero-point energies and entropies of $\mathrm{HOO}^{*}$ and non-adsorbed gas-phase molecules were adopted from the previous literatures.[14,33] As the ground state of $\mathrm{O}_{2}$ molecule is poorly described in DFT calculations, we used gas-phase $\mathrm{H}_{2} \mathrm{O}$ and $\mathrm{H}_{2}$ as reference states as they are readily treated in the DFT calculations. Please noted that we also calculated the $\Delta G_{\mathrm{HOO}^{*}}$ by using the plane-wave projector-augmented wave method as implemented in the Vienna ab initio simulation (VASP) package[34,35], and the results show that the difference of calculated values by using $\mathrm{Dmol}^{3}$ and VASP are less than $0.06 \mathrm{eV}$, as shown in Fig. S2 in ESI.

\section{Conflicts of interest}

There are no conflicts of interest to declare.

\section{Acknowledgements}

This research was undertaken with the assistance of resources provided by the National Computing Infrastructure $(\mathrm{NCl})$ facility at the Australian National University; allocated through both the National Computational Merit Allocation Scheme supported by the Australian Government and the Australian Research Council grant LE160100051 (Maintaining and enhancing meritbased access to the NCI National Facility, 2016-2018). 


\section{Notes and references}

1 J. M. Campos-Martin, G. Blanco-Brieva and J. L. G. Fierro, Angew. Chem. Int. Ed., 2006, 45, 6962-6984.

2 C. Samanta, Appl. Catal., A, 2008, 350, 133-149.

3 R. Hage and A. Lienke, Angew. Chem. Int. Ed., 2006, 45, 206222.

4 www.gminsights.com/pressrelease/hydrogen-peroxidemarket

5 J. K. Edwards, B. Solsona, E. N. N, A. F. Carley, A. A. Herzing, C. J. Kiely and G. J. Hutchings, Science, 2009, 323, 1037-1041.

6 B. E. Solsona, J. K. Edwards, P. Landon, A. F. Carley, A. Herzing, C. J. Kiely and G. J. Hutchings, Chem. Mater., 2006, 18, 2689-2695.

7 S. J. Freakley, Q. He, J. H. Harrhy, L. Lu, D. A. Crole, D. J. Morgan, E. N. Ntainjua, J. K. Edwards, A. F. Carley, A. Y. Borisevich, C. J. Kiely and G. J. Hutchings, Science, 2016, 351, 965-968.

8 J. K. Edwards, S. J. Freakley, R. J. Lewis, J. C. Pritchard and G. J. Hutchings, Catal. Today, 2015, 248, 3-9.

9 R. B. Rankin and J. Greeley, ACS Catal., 2012, 2, 2664-2672.

10 K. N. Wood, R. O'Hayre and S. Pylypenko, Energy Environ. Sci., 2014, 7, 1212-1249.

11 P. Drogui, S. Elmaleh, M. Rumeau, C. Bernard and A. Rambaud, J. Appl. Electrochem., 2001, 31, 877-882.

12 I. Yamanaka and T. Murayama, Angew. Chem. Int. Ed., 2008, 47, 1900-1902.

13 Z. W. Seh, J. Kibsgaard, C. F. Dickens, I. Chorkendorff, J. K. Nørskov and T. F. Jaramillo, Science, 2017, 355, eaad4998.

14 S. Siahrostami, A. Verdaguer-Casadevall, M. Karamad, D. Deiana, P. Malacrida, B. Wickman, M. Escudero-Escribano, E. A. Paoli, R. Frydendal, T. W. Hansen, I. Chorkendorff, I. E. L. Stephens and J. Rossmeisl, Nat. Mater., 2013, 12, 1137-1143

15 A. Verdaguer-Casadevall, D. Deiana, M. Karamad, S. Siahrostami, P. Malacrida, T. W. Hansen, J. Rossmeisl, I. Chorkendorff and I. E. L. Stephens, Nano Lett., 2014, 14, 1603-1608.

16 J. S. Jirkovský, I. Panas, E. Ahlberg, M. Halasa, S. Romani and D. J. Schiffrin, J. Am. Chem. Soc., 2011, 133, 1943219441.

17 C. Hu and L. Dai, Angew. Chem. Int. Ed., 2016, 55, 1173611758.

18 M. Melchionna, P. Fornasiero and M. Prato, Adv. Mater., 2018, 1802920.

19 H. W. Kim, M. B. Ross, N. Kornienko, L. Zhang, J. Guo, P. Yang and B. D. McCloskey, Nat. Catal., 2018, 1, 282-290.

20 A. Wang, J. Li and T. Zhang, Nat. Rev. Chem., 2018, 2, 65-81.

21 C. R. A. Catlow, S. A. French, A. A. Sokol and J. M. Thomas, Philos. Trans. Ser. A Math. Phys. Eng. Sci., 2005, 363, 913936.

22 J. Zhang, Z. Zhao, Z. Xia and L. Dai, Nat. Nanotechnol., 2015, 10, 444-452.

23 Y. Jiao, Y. Zheng, M. Jaroniec and S. Z. Qiao, J. Am. Chem. Soc., 2014, 136, 4394-4403.

24 M. Acik, G. Lee, C. Mattevi, M. Chhowalla, K. Cho and Y. J. Chabal, Nat. Mater., 2010, 9, 840-845.

25 M. Acik, G. Lee, C. Mattevi, A. Pirkle, R. M. Wallace, M. Chhowalla, K. Cho and Y. Chabal, J. Phys. Chem. C, 2011, 115, 19761-19781.

26 V. Viswanathan, H. A. Hansen, J. Rossmeisl and J. K. Nørskov, J. Phys. Chem. Lett., 2012, 3, 2948-2951.

27 B. Delley, J. Chem. Phys., 2000, 113, 7756-7764.

28 J. P. Perdew and Y. Wang, Phys. Rev. B, 1992, 45, 1324413249.

29 F. Ortmann, F. Bechstedt and W. G. Schmidt, Phys. Rev. B, 2006, 73, 205101.

30 M. R. Rezapour, J. Yun, G. Lee and K. S. Kim, J. Phys. Chem. Lett., 2016, 7, 5049-5055.
31 H. J. Monkhorst and J. D. Pack, Phys. Rev. B, 1976, 13, 51885192.

32 J. K. Nørskov, T. Bligaard, A. Logadottir, J. R. Kitchin, J. G. Chen, S. Pandelov and U. Stimming, J. Electrochem. Soc., 2005, 152, J23-J26.

33 J. K. Nørskov, J. Rossmeisl, A. Logadottir, L. Lindqvist, J. R. Kitchin, T. Bligaard and H. Jónsson, J. Phys. Chem. $B, 2004,108,17886-17892$.

34 G. Kresse and J. Hafner, Phys. Rev. B, 1994, 49, 1425114269.

35 G. Kresse and D. Joubert, Phys. Rev. B, 1999, 59, 1758-1775. 